\title{
Monthly Electric Energy Consumption Forecasting Using Multiwindow Moving Average and Hybrid Growth Models
}

\author{
Ming Meng, ${ }^{1,2}$ Wei Shang, ${ }^{3}$ and Dongxiao Niu ${ }^{1}$ \\ ${ }^{1}$ School of Economics and Management, North China Electric Power University, No. 619, Yonghua Street, Baoding, Hebei 071003, China \\ ${ }^{2}$ Soft Science Research Base of Hebei Province, North China Electric Power University, Baoding, Hebei 071003, China \\ ${ }^{3}$ School of Economics, Hebei University, Baoding, Hebei 071002, China \\ Correspondence should be addressed to Ming Meng; ncepumm@126.com
}

Received 24 December 2013; Accepted 5 May 2014; Published 15 May 2014

Academic Editor: Shan Zhao

Copyright (C) 2014 Ming Meng et al. This is an open access article distributed under the Creative Commons Attribution License, which permits unrestricted use, distribution, and reproduction in any medium, provided the original work is properly cited.

\begin{abstract}
Monthly electric energy consumption forecasting is important for electricity production planning and electric power engineering decision making. Multiwindow moving average algorithm is proposed to decompose the monthly electric energy consumption time series into several periodic waves and a long-term approximately exponential increasing trend. Radial basis function (RBF) artificial neural network (ANN) models are used to forecast the extracted periodic waves. A novel hybrid growth model, which includes a constant term, a linear term, and an exponential term, is proposed to forecast the extracted increasing trend. The forecasting results of the monthly electric energy consumption can be obtained by adding the forecasting values of each model. To test the performance by comparison, the proposed and other three models are used to forecast China's monthly electric energy consumption from January 2011 to December 2012. Results show that the proposed model exhibited the best performance in terms of mean absolute percentage error (MAPE) and maximal absolute percentage error (MaxAPE).
\end{abstract}

\section{Introduction}

Electric energy consumption is essential for promoting economic development and raising people's living standard [14]. In contrast to other energy sources, electric energy cannot be stored for large-scale consumption. From an economic viewpoint, the supply and demand of electric energy must be balanced at any given time. Therefore, a precise forecasting of electric energy consumption is very important for the economic operation of an electric power system. Studies on electric energy consumption forecasting are usually divided into two categories on the basis of forecasting indicators: short-term [5-8] and mid-/long-term forecasting [9-11]. The current study fits into the second category because monthly electric energy consumption forecasting is an important basis for electricity production planning and electric power engineering decision making.

Researchers have developed two ideas for mid-/long-term electric energy consumption forecasting. One idea focuses on the relationship between electric energy consumption and its influencing factors. Models originating from this idea usually formulate an equation to quantitatively simulate such relationship. These models obtain the forecasting results by inputting the values of the influencing factors during the forecasting period into the equation [12-14]. Most of these models exhibit satisfactory performance in simulating historical data. However, the exact values of the influencing factors during the forecasting period are difficult to determine; therefore, these models usually cannot produce satisfactory results when used for forecasting. The other idea concentrates on the changing rules of the electric energy consumption trend itself. Related models use the historical electricity consumption data to formulate trend simulation equations and obtain the forecasting results by means of trend extrapolation [15-17]. The forecasting model proposed in the current study belongs to the latter category.

The difficulty in forecasting monthly electric energy consumption by means of trend extrapolation lies in the complexity of the consumption curve. The consumption curve generally includes two subtrends. First, a long-term increasing trend typically exists in most cases. Basic electric energy consumption usually increases with the development 
of social economy. Second, periodic waves always exist, as a result of the effects of people's living habits and work styles according to alternating seasons. Therefore, the presence of both a long-term increasing trend and periodic waves with different frequencies and amplitudes increases the difficulties in trend extrapolation.

The artificial neural network (ANN) is a traditional nonlinear trend extrapolation model. The primary advantage of ANN is its capability of modeling nonlinear relations without the supervision of human experts [18, 19]. When ANN is used for forecasting by means of trend extrapolation, several continuous electric energy consumption data would be selected as input, and the output would be the forecasting result. However, the very complex nature of the monthly electric energy consumption curve greatly affects the generalization ability of the ANN. That is, when used to forecast the monthly electric energy consumption, ANN produces satisfactory simulation results but results in poor forecasting precision. The key idea in improving forecasting precision is to simplify the monthly electric energy consumption curve by means of trend extraction.

Zhao and Wei [20] conducted a study on the trend extraction problem and proposed several applicable methods. González-Romera et al. [21-23] have tested these methods and recommended the moving average as their first choice. They used a moving average algorithm with a 12-month window width to decompose the monthly electric energy consumption time series into a wave curve and a longterm increasing curve. The extracted wave and increasing curves were separately forecasted by Fourier series and ANN models. The forecasting results of the monthly electric energy consumption were obtained by adding the forecasting results of the extracted two curves. Their models have been proven to be better than time series $[24,25]$ and single ANN models.

Meng et al. [26] pointed out that this model still has at least two flaws. First, the extracted wave trend is still very complex, containing several subwave trends with different frequencies and amplitudes. For this reason, GonzálezRomera et al. [21-23] selected the Fourier series and not ANN to forecast the wave curve. However, the Fourier series is limited by the algorithm itself, such that it can only simulate waves with invariable amplitudes. In fact, the amplitudes of periodic waves extracted from the monthly electric energy consumption time series usually increase gradually with the development of social economy. Second, the extracted increasing curve is usually not very smooth because of the effects of changes in the external environment (e.g., economic crisis, political unrest, and unpredictable weather conditions). Using ANN to forecast this curve tends to result in large forecasting errors. For these reasons, Meng et al. [26] adopted wavelet transform to decompose the monthly electric energy consumption time series into a long-term increasing curve and several simple wave curves. They used ANN to forecast the extracted wave curves and selected the GM $(1,1)$ [27-29], which is a widely used smallsample exponential trend extrapolating model, to forecast the extracted long-term increasing curve, which usually presents an approximately exponential trend. The forecasting results of the monthly electric energy consumption were obtained by adding the forecasting results of all the extracted curves.

Nevertheless, the forecasting model proposed by Meng et al. [26] also has its limitations. The electric energy consumption trend has numerous waves with different frequencies and amplitudes as a result of the effects of the people's changing living habits and work styles according to alternating seasons; therefore, the least common multiple of all the periods of waves should be 12 months. Thus, the wave period may be $12,6,4,3$, or 2 months. However, limited by the algorithm itself, the period of waves extracted by wavelet transform could only exist in multiples. That is, the period of waves could be 12, 6 , and 3 months. Therefore, the algorithm cannot cover all the possible periods. Moreover, in essence, the forecasting equation of $\operatorname{GM}(1,1)[30]$ is a homogeneous exponential curve, which is simply a special form of the approximately exponential curve. GM $(1,1)$ is suitable only for a time series with a steadily increasing rate. This characteristic does not necessarily apply to the extracted long-term increasing curve of the monthly electric energy consumption time series.

Therefore, the present study proposes a multiwindow moving average algorithm to obtain accurate extracted results of the wave curves. This algorithm can extract wave curves under all the possible periods. Furthermore, a hybrid growth model is proposed to improve the forecasting results of the extracted long-term increasing curve. A constant term is added to the forecasting equation of $\operatorname{GM}(1,1)$, as well as a linear term, because the approximate exponential trend of the extracted long-term increasing curve is unstable and a linear trend may appear in special periods.

\section{Novel Forecasting Models}

2.1. Multiwindow Moving Average Algorithm. The moving average algorithm is written as follows:

$$
T_{i}=\frac{1}{n}\left[C_{i}+C_{i-1}+\cdots+C_{i-(n-1)}\right],
$$

where $T_{i}$ is a smoothed datum, $C_{i}$ is a datum of the original time series, and $n$ is the window size. Aside from the longterm increasing trend, time series $T$ contains the periodic waves, wherein periods are multiples of $n$.

Let

$$
R_{i}=C_{i}-T_{i}
$$

be the remained datum; then time series $R$ would present a wave curve with a period of $n$.

Figure 1 shows the process of the multiwindow moving average algorithm.

The left branch of Figure 1 is selected as an example to explain the multiwindow moving average process. As shown in Figure 1, the original time series (denoted by OTS) is proposed by a moving average algorithm with a window size of 2 (denoted by W2). Then, the smoothed time series (denoted by S2) and the remained time series (denoted by R2) are obtained. By using a moving average algorithm with a window size of 4 to process S2, the smoothed time series 


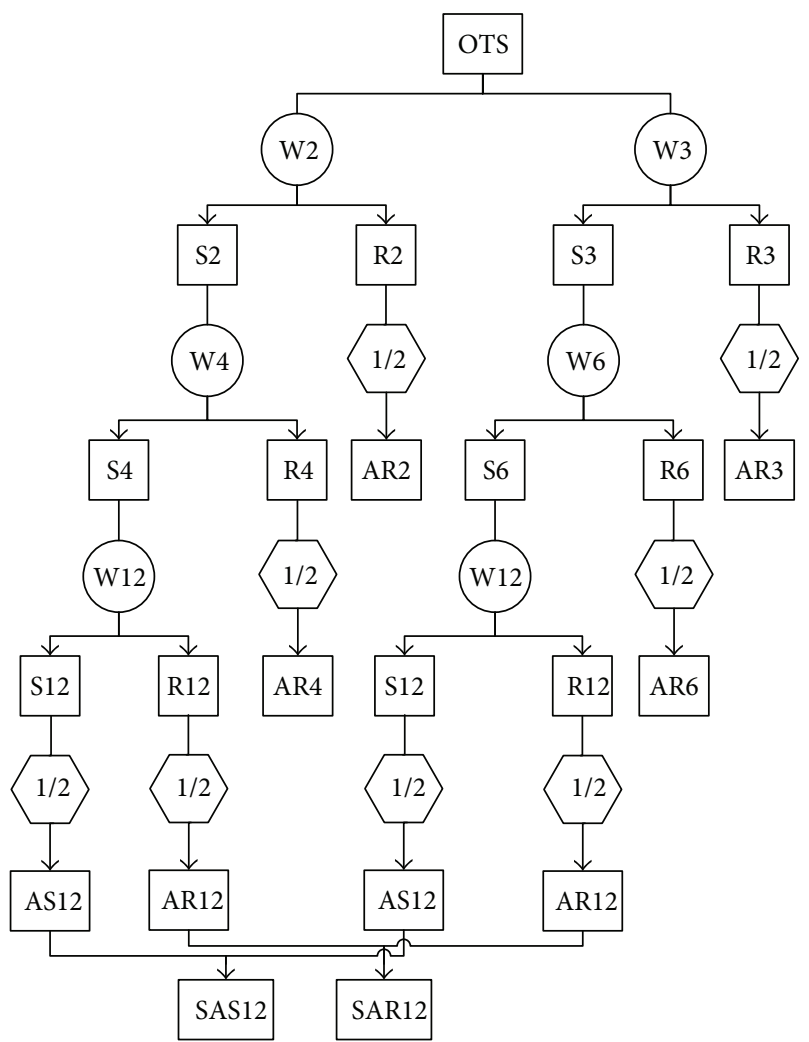

FIgURE 1: Process of the multiwindow moving average algorithm.

(denoted by S4) and the remained time series (denoted by R4) are obtained. By using a similar algorithm to process $\mathrm{S} 4$, the S12 and R12 time series are also obtained. By following the decomposition process shown in the right branch of Figure 1, R3, R6, R12, and S12 are also obtained.

As the OTS are decomposed twice, R2, R3, .., R12 and S12 are multiplied by $1 / 2$ and renamed as AR2, AR3,.., AR12 and AS12 to ensure that the summation of all the extracted time series is equal to the OTS. Furthermore, although the left and right branches can both obtain AS12 and AR12, the two AS12 and two AR12 should be summed separately. Accordingly, the results are named as SAS12 and SAR12, respectively.

The extracted time series are AR2, AR3, AR4, AR6, SAR12, and SAS12. The sum of all the extracted time series is equal to the OTS. As the maximum period of all the waves in monthly electric energy consumption time series is 12 , time series SAS12 should have no periodic waves and should thus be a long-term increasing trend. Other time series are wave trends with different periods.

2.2. Hybrid Growth Model. The forecasting/simulation equation of GM $(1,1)$ is usually written as follows:

$$
\begin{array}{r}
x(k+1)=(1-\exp (a))\left(x(1)-\frac{u}{a}\right) \exp (-a k), \\
k=0,1,2, \ldots,
\end{array}
$$

where $x(k)$ is the $k$ th value of the time series and $a$ and $u$ are the parameters.
Clearly, (3) has no constant term because it is a homogeneous exponential equation.

Equation (3) can be written as follows:

$$
\begin{array}{r}
x(k+1) \\
=(1-\exp (a))\left(x(1)-\frac{u}{a}\right) \exp (-a) \exp [-a(k-1)], \\
k=0,1,2, \ldots .
\end{array}
$$

$$
\begin{aligned}
& \text { If } \lambda_{1}=\exp (-a) \text {, then (4) can be further written as follows: } \\
& \qquad x(k+1)=\lambda_{1} x(k) .
\end{aligned}
$$

The hybrid growth equation, which includes a constant term, a linear term, and an exponential term, can then be written as follows:

$$
x(k+1)=\lambda_{1} x(k)+\lambda_{2} k+\lambda_{3} .
$$

Given that $n$ time series data exist, for each group of estimated parameters, (6) can be written as follows:

$$
\begin{aligned}
x(2) & =\widehat{\lambda}_{1} x(1)+\widehat{\lambda}_{2}+\widehat{\lambda}_{3}+\varepsilon_{1}, \\
x(3) & =\widehat{\lambda}_{1} x(2)+2 \widehat{\lambda}_{2}+\widehat{\lambda}_{3}+\varepsilon_{2}, \\
& \vdots \\
x(n) & =\widehat{\lambda}_{1} x(n-1)+(n-1) \hat{\lambda}_{2}+\widehat{\lambda}_{3}+\varepsilon_{n-1},
\end{aligned}
$$

where $\varepsilon$ is the residual.

If

$$
\begin{gathered}
\mathbf{Y}=\left[\begin{array}{c}
x(2) \\
x(3) \\
\vdots \\
x(n)
\end{array}\right], \quad \mathbf{B}=\left[\begin{array}{ccc}
x(1) & 1 & 1 \\
x(2) & 2 & 1 \\
\vdots & \vdots & \vdots \\
x(n-1) & n-1 & 1
\end{array}\right], \\
\hat{\lambda}=\left[\begin{array}{c}
\hat{\lambda}_{1} \\
\hat{\lambda}_{2} \\
\hat{\lambda}_{3}
\end{array}\right], \quad \boldsymbol{\varepsilon}=\left[\begin{array}{c}
\varepsilon_{1} \\
\varepsilon_{2} \\
\vdots \\
\varepsilon_{n-1}
\end{array}\right],
\end{gathered}
$$

then (7) can be written as follows:

$$
\mathbf{Y}=\mathbf{B} \widehat{\lambda}+\varepsilon
$$

To obtain the optimal parameter estimations, the residual sum of squares must be minimized.

Let

$$
\begin{aligned}
Q & =\boldsymbol{\varepsilon}^{\prime} \boldsymbol{\varepsilon} \\
& =(\mathbf{Y}-\mathbf{B} \hat{\boldsymbol{\lambda}})^{\prime}(\mathbf{Y}-\mathbf{B} \hat{\boldsymbol{\lambda}}) \\
& =\mathbf{Y}^{\prime} \mathbf{Y}-\mathbf{Y}^{\prime} \mathbf{B} \hat{\boldsymbol{\lambda}}-\hat{\lambda}^{\prime} \mathbf{B}^{\prime} \mathbf{Y}+\hat{\lambda}^{\prime} \mathbf{B}^{\prime} \mathbf{B} \hat{\lambda}
\end{aligned}
$$


To obtain the minimum of $Q$, its derivative with respect to the parameter vector must be equal to 0 . Consider

$$
\frac{\partial Q}{\partial \widehat{\lambda}}=-2 \mathbf{B}^{\prime} \mathbf{Y}+2 \mathbf{B}^{\prime} \mathbf{B} \widehat{\lambda}=0 .
$$

Then,

$$
\widehat{\lambda}=\left(\mathbf{B}^{\prime} \mathbf{B}\right)^{-1} \mathbf{B}^{\prime} \mathbf{Y}
$$

Up to this point, the optimal parameter estimations of (6) can be obtained.

However, (6) is an iterative equation. To obtain the optimal starting point of iteration, let

$$
\widehat{x}(1)=x(1)+\widehat{\lambda}_{4}
$$

The optimal estimation of $\lambda_{4}$ is obtained by

$$
\min _{\widehat{\beta}_{4}} \sum_{k=1}^{n}[\widehat{x}(k)-x(k)]^{2} .
$$

Equation (14) has been proven to be a nonconstrained optimization, and its solution [31] is

$$
\begin{aligned}
& \widehat{\beta}_{4} \\
& =\left(\sum_{k=1}^{n-1}\left[x(k+1)-\beta_{1}^{k} x(1)-\beta_{2} \sum_{j=1}^{k} j \beta_{1}^{k-1}-\frac{1-\beta_{1}^{k}}{1-\beta_{1}} \beta_{3}\right] \beta_{1}^{k}\right) \\
& \times\left(1+\sum_{k=1}^{n-1}\left(\beta_{1}^{k}\right)^{2}\right)^{-1} .
\end{aligned}
$$

Thus, the hybrid growth model, which is used to forecast the extracted long-term increasing trend, is completed.

2.3. Forecasting Process. The above models can be used for both one-step and multistep ahead forecastings. As the former is used more often, and especially useful for the production operation management of the power-generation companies, this paper designs the one-step ahead forecasting process as follows.

(A) Curve Extraction. The aforementioned multiwindow moving average algorithm is used to decompose the OTS of the monthly electric energy consumption. A long-term increasing curve and several wave curves with different periods are obtained.

(B) Subtrend Forecasting. The aforementioned hybrid growth model is used to forecast the extracted long-term increasing curve. For each forecasting point, continuous 12 data preceding it are used to estimate the equation parameters $\left(\lambda_{1}, \lambda_{2}\right.$, $\lambda_{3}$, and $\lambda_{4}$ ). If $k=12$ in (6), then the forecasting result could be obtained.

The radial basis function (RBF) ANN model is used to forecast the extracted wave curves. Similar to [26], for each forecasting point in an extracted wave curve, 60 consecutive

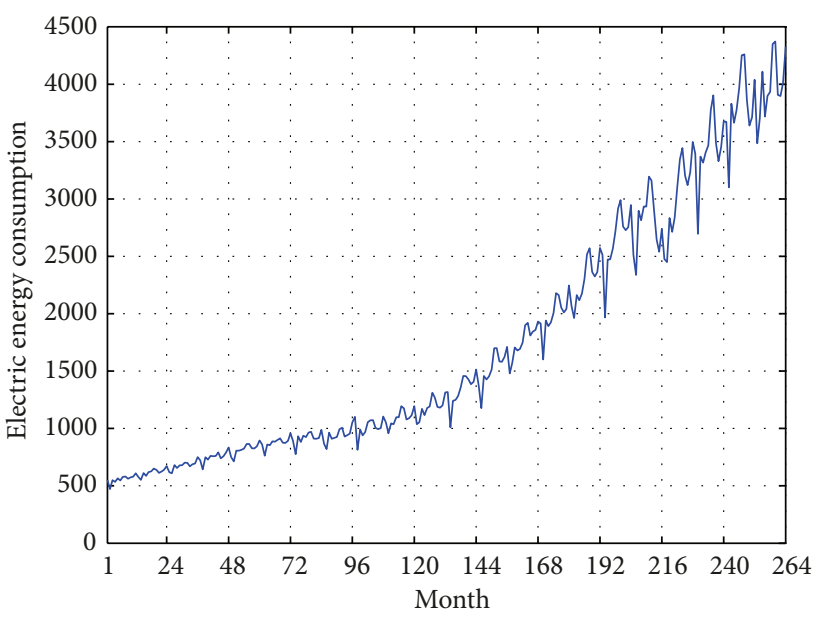

FIGURE 2: Monthly electric energy consumption curve of China.

previous values of it were selected for RBF ANN training. The 13 th to 60th values were taken as outputs. For each output value, 12 previous values of it were selected as the inputs. After training, the ANN is used for forecasting. Based on the same rule, when forecasting the $j$ th point, the 12 consecutive previous values of it are entered and the output of $\mathrm{ANN}$ is considered the forecasting result.

(C) Monthly Electric Energy Consumption. The forecasting result of monthly electric energy consumption is obtained by adding the forecasting results of each extracted curve.

\section{Model Test}

3.1. Data Selection and Preliminary Analysis. At present, China is the largest electric energy consumer in the world. In 2012, its global consumption share reached 21.94\% [32]. In this section, the monthly electric energy consumption of China was used to validate the proposed model. Figure 2 shows the consumption curve (in $10^{8} \mathrm{~kW} \cdot \mathrm{h}$ ) from January 1991 to December 2012.

As shown in Figure 2, China's monthly electric energy consumption curve contains a long-term approximately exponential increasing trend and several periodic wave trends. Clearly, the amplitudes of the periodic wave trends increase gradually, thus requiring the use of ANN to forecast these wave trends.

The monthly electric energy consumption curve of China was decomposed to several subcurves by using the aforementioned multiwindow moving average algorithm. Figure 3 shows the decomposed results from January 1993 to December 2012 .

The SAS12 in Figure 3 has no wave trends. This finding verifies that the maximum period of all periodic waves is 12. Furthermore, the increasing trend of SAS12 is unstable and near a linear trend in special periods (this characteristic can also be found in Figure 2), thus implying the necessity of using the hybrid growth model in forecasting the increasing trend. 
TABLE 1: Real data and forecasting results.

\begin{tabular}{|c|c|c|c|c|c|}
\hline Month & $\mathrm{RD}$ & M1 & M2 & M3 & M4 \\
\hline January 2011 & 3672.1 & 3811.3 & 3402 & 3677.8 & 3642.3 \\
\hline February & 3100.8 & 3545.7 & 3347.4 & 3672.1 & 3672.5 \\
\hline March & 3830.1 & 3722 & 3863.9 & 3100.8 & 3277.6 \\
\hline April & 3663.8 & 3817.8 & 3826.2 & 3830.1 & 3838.4 \\
\hline May & 3775.4 & 3841.6 & 3837 & 3663.8 & 3465.2 \\
\hline June & 3968.2 & 3860 & 3863.5 & 3775.4 & 3818 \\
\hline July & 4251.5 & 4062.2 & 4106.7 & 3968.2 & 3875.7 \\
\hline August & 4260.4 & 4066.6 & 4099.7 & 4251.5 & 4116.1 \\
\hline September & 3860.6 & 3694.3 & 3783.7 & 4260.4 & 4244.3 \\
\hline October & 3640.4 & 3669.4 & 3636.1 & 3860.6 & 4124.5 \\
\hline November & 3713 & 3886.3 & 3724.6 & 3640.4 & 3904.2 \\
\hline December & 4038.1 & 3967.6 & 3939.6 & 3713 & 3793.3 \\
\hline January 2012 & 3485 & 3963 & 3909 & 4038.1 & 3983.8 \\
\hline February & 3702 & 3454.6 & 3597.3 & 3485 & 3624.5 \\
\hline March & 4109 & 3966 & 3882.4 & 3702 & 3822.5 \\
\hline April & 3718 & 3953.7 & 4174.9 & 4109 & 3913.2 \\
\hline May & 3898 & 3999.8 & 4013 & 3718 & 3720 \\
\hline June & 3934 & 4004.5 & 4071.4 & 3898 & 3990.4 \\
\hline July & 4351 & 4175 & 4415.3 & 3934 & 3869.8 \\
\hline August & 4373 & 4222.6 & 4607.5 & 4351 & 4188.2 \\
\hline September & 3907.3 & 4158.1 & 4438.2 & 4373 & 4285.1 \\
\hline October & 3897.7 & 3972.3 & 4403.3 & 3907.3 & 4194.5 \\
\hline November & 4010.5 & 4137.2 & 4613 & 3897.7 & 4123.7 \\
\hline December & 4327.2 & 4277.4 & 4682.8 & 4010.5 & 4015.4 \\
\hline
\end{tabular}

3.2. Results and Discussions. To evaluate the performance by comparison, the forecasting model proposed in this paper (denoted by M1), model used in [26] (denoted by M2), single RBF ANN model (denoted by M3), and ARIMA model (denoted by M4) were all used to forecast China's monthly electric energy consumption from January 2011 to December 2012. For the single RBF ANN, the training and forecasting rules were the same as those in forecasting the extracted wave curves. For the ARIMA model, the first order difference was used to obtain a stationary time series. According to the autocorrelation function and partial autocorrelation function analysis to the difference time series, the numbers of autoregressive term and moving average term are all set to 2 . Similar to the RBF ANN model, for each forecasting point in an extracted wave curve, 60 consecutive previous values of it were selected to build the ARIMA model. Real data (denoted by RD) and forecasting results of the four models are listed in Table 1.

To evaluate the forecasting performance of each model by comparison, the mean absolute percentage error (MAPE, in $\%$ ) and the maximal absolute percentage error (MaxAPE, in $\%)[26,33]$ were used as indicators of forecasting precision. Consider

$$
\operatorname{MAPE}=\operatorname{mean}_{i}\left(\left|\frac{x(i)-\widehat{x}(i)}{x(i)}\right|\right) \cdot 100,
$$

TABLE 2: Forecasting errors of each model.

\begin{tabular}{lcccc}
\hline & M1 & M2 & M3 & M4 \\
\hline MAPE & 4.37 & 5.53 & 6.81 & 7.26 \\
MaxAPE & 14.35 & 15.02 & 19.04 & 18.44 \\
\hline
\end{tabular}

where $x(i)$ is the electric energy consumption value of the $i$ th month, $\widehat{x}(i)$ represents its forecasting result. Consider

$$
\operatorname{MaxAPE}=\max _{i}\left(\left|\frac{x(i)-\widehat{x}(i)}{x(i)}\right|\right) \cdot 100 .
$$

Despite having similar functions of determining which is the best model, these two error analysis indicators have fine distinctions. MAPE is the most widely used indicator of accuracy, because this indicator reflects the general closeness of the forecasting results to RD. MaxAPE generates the worst forecasting result and reflects the maximal forecasting risk.

By using the data in Table 1 and (16) and (17), the results of each error analysis indicator were obtained. These values (in \%) are listed in Table 2.

On the whole, as the trend extraction algorithms simplified the forecasting curves, M1 and M2 are better than M3 and M4 for each indicator. As the period of the extracted waves of $\mathrm{M} 1$ is more reasonable than $\mathrm{M} 2$ and the hybrid growth model is more applicable to the extracted long-term increasing curve than $\operatorname{GM}(1,1), \mathrm{M} 1$ is always better than 


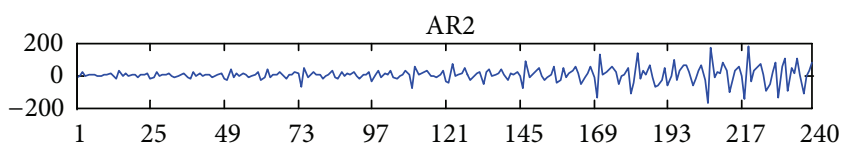

(a)

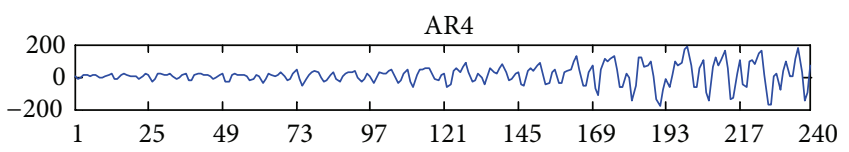

(c)

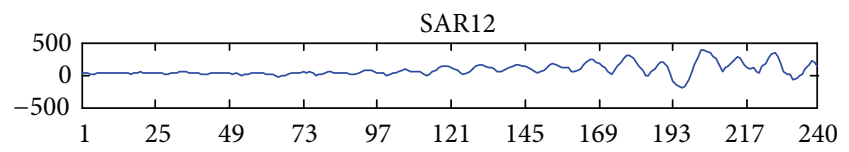

(e)

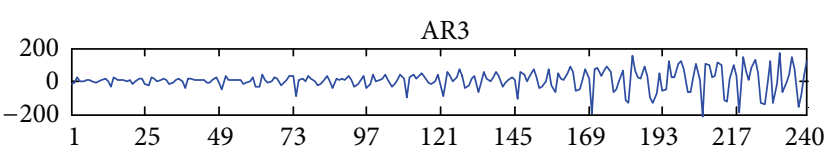

(b)

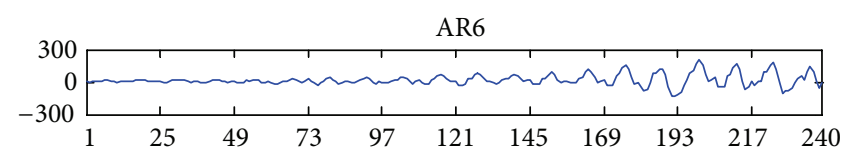

(d)

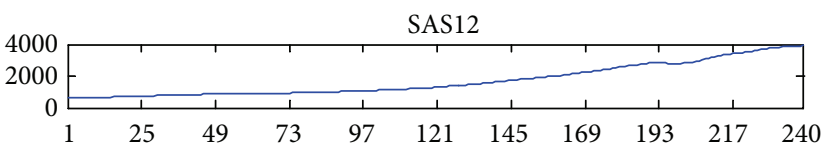

(f)

Figure 3: Trends of extracted curves from January 1993 to December 2012.

M2 for each indicator. Especially for MAPE, which is usually considered the most important indicator, M1 (4.37\%) is much smaller than M2 (5.53\%).

\section{Conclusions}

Monthly electric energy consumption time series usually has two characteristics. First, the main trend of the said time series presents an approximately exponential increasing feature. Second, the period of the wave trends can only be 12, $6,4,3$, or 2 months. Thus, this study proposed a multiwindow moving average algorithm to decompose the said time series and a novel hybrid growth model to forecast the extracted long-term increasing trend. To evaluate the performance by comparison, the proposed and other three models were used to forecast China's monthly electric energy consumption from January 2011 to December 2012. Empirical results show that, because of the aforementioned innovations, the MAPE and MaxAPE of the proposed model are smaller than those of the other three models.

\section{Conflict of Interests}

The authors declare that there is no conflict of interests regarding the publication of this paper.

\section{Acknowledgments}

This study was supported by the National Natural Science Foundation of China (NSFC) (71201057 and 71071052) and the Fundamental Research Funds for the Central Universities (2014MS148).

\section{References}

[1] A. E. Akinlo, "Electricity consumption and economic growth in Nigeria: evidence from cointegration and co-feature analysis," Journal of Policy Modeling, vol. 31, no. 5, pp. 681-693, 2009.
[2] S.-H. Yoo and S.-Y. Kwak, "Electricity consumption and economic growth in seven South American countries," Energy Policy, vol. 38, no. 1, pp. 181-188, 2010.

[3] M. Shahbaz and H. H. Lean, "The dynamics of electricity consumption and economic growth: a revisit study of their causality in Pakistan," Energy, vol. 39, no. 1, pp. 146-153, 2012.

[4] H. Gurgul and $Ł$. Lach, "The electricity consumption versus economic growth of the Polish economy," Energy Economics, vol. 34 , no. 2, pp. 500-510, 2012.

[5] L. J. Soares and M. C. Medeiros, "Modeling and forecasting short-term electricity load: a comparison of methods with an application to Brazilian data," International Journal of Forecasting, vol. 24, no. 4, pp. 630-644, 2008.

[6] H. Kebriaei, B. N. Araabi, and R.-K. Ashkan, "Short-term load forecasting with a new nonsymmetric penalty function," IEEE Transactions on Power Systems, vol. 26, no. 4, pp. 1817-1825, 2011.

[7] D. Niu, H. Shi, and D. D. Wu, "Short-term load forecasting using bayesian neural networks learned by Hybrid Monte Carlo algorithm," Applied Soft Computing Journal, vol. 12, no. 6, pp. 1822-1827, 2012.

[8] Y. Yang, J. Wu, Y. Chen, and C. Li, "A new strategy for shortterm load forecasting," Abstract and Applied Analysis, vol. 2013, Article ID 208964, 9 pages, 2013.

[9] O. A. S. Carpinteiro, R. C. Leme, A. C. Z. Souza, C. A. M. Pinheiro, and E. M. Moreira, "Long-term load forecasting via a hierarchical neural model with time integrators," Electric Power Systems Research, vol. 77, no. 3-4, pp. 371-378, 2007.

[10] X. Liu and J. Fang, "Long-term load forecasting based on a time-variant ratio multiobjective optimization fuzzy time series model," Mathematical Problems in Engineering, vol. 2013, Article ID 781043, 7 pages, 2013.

[11] L. Ghelardoni, A. Ghio, and D. Anguita, "Energy load forecasting using empirical mode decomposition and support vector regression," IEEE Transactions on Smart Grid, vol. 4, no. 1, pp. 549-556, 2013.

[12] M. Meng and D. Niu, "Annual electricity consumption analysis and forecasting of China based on few observations methods," Energy Conversion and Management, vol. 52, no. 2, pp. 953-957, 2011. 
[13] J. F. M. Pessanha and N. Leon, "Long-term forecasting of household and residential electric customers in Brazil," IEEE Latin America Transactions, vol. 10, no. 2, pp. 1537-1543, 2012.

[14] Á. L. Santana, G. B. Conde, L. P. Rego et al., "PREDICTdecision support system for load forecasting and inference: a new undertaking for Brazilian power suppliers," International Journal of Electrical Power \& Energy Systems, vol. 38, no. 1, pp. 33-45, 2012.

[15] P. Zhou, B. W. Ang, and K. L. Poh, "A trigonometric grey prediction approach to forecasting electricity demand," Energy, vol. 31, no. 14, pp. 2839-2847, 2006.

[16] A. Azadeh, S. F. Ghaderi, and S. Sohrabkhani, "Annual electricity consumption forecasting by neural network in high energy consuming industrial sectors," Energy Conversion and Management, vol. 49, no. 8, pp. 2272-2278, 2008.

[17] Y. Wang, J. Wang, G. Zhao, and Y. Dong, "Application of residual modification approach in seasonal ARIMA for electricity demand forecasting: a case study of China," Energy Policy, vol. 48, pp. 284-294, 2012.

[18] M. T. Hagan, H. B. Demuth, and M. H. Beale, Neural Network Design, PWS Publishing, Boston, Mass, USA, 1996.

[19] S. Haykin, Neural Networks: A Comprehensive Foundation, Pearson Education, Upper Saddle River, NJ, USA, 2009.

[20] S. Zhao and G. W. Wei, "Jump process for the trend estimation of time series," Computational Statistics \& Data Analysis, vol. 42, no. 1-2, pp. 219-241, 2003.

[21] E. González-Romera, M. A. Jaramillo-Morán, and D. CarmonaFernández, "Monthly electric energy demand forecasting based on trend extraction," IEEE Transactions on Power Systems, vol. 21, no. 4, pp. 1946-1953, 2006.

[22] E. González-Romera, M. A. Jaramillo-Morán, and D. CarmonaFernández, "Forecasting of the electric energy demand trend and monthly fluctuation with neural networks," Computers \& Industrial Engineering, vol. 52, no. 3, pp. 336-343, 2007.

[23] E. González-Romera, M. A. Jaramillo-Morán, and D. CarmonaFernández, "Monthly electric energy demand forecasting with neural networks and Fourier series," Energy Conversion and Management, vol. 49, no. 11, pp. 3135-3142, 2008.

[24] R. E. Abdel-Aal and A. Z. Al-Garni, "Forecasting monthly electric energy consumption in Eastern Saudi Arabia using univariate time-series analysis," Energy, vol. 22, no. 11, pp. 10591069, 1997.

[25] S. Saab, E. Badr, and G. Nasr, "Univariate modeling and forecasting of energy consumption: the case of electricity in Lebanon," Energy, vol. 26, no. 1, pp. 1-14, 2001.

[26] M. Meng, D. Niu, and W. Sun, "Forecasting monthly electric energy consumption using feature extraction," Energies, vol. 4, no. 10, pp. 1495-1507, 2011.

[27] I. J. Lu, C. Lewis, and S. J. Lin, "The forecast of motor vehicle, energy demand and $\mathrm{CO}_{2}$ emission from Taiwan's road transportation sector," Energy Policy, vol. 37, no. 8, pp. 29522961, 2009.

[28] M. Mao and E. C. Chirwa, "Application of grey model GM(1, 1) to vehicle fatality risk estimation," Technological Forecasting and Social Change, vol. 73, no. 5, pp. 588-605, 2006.

[29] C.-S. Lin, F.-M. Liou, and C.-P. Huang, "Grey forecasting model for $\mathrm{CO}_{2}$ emissions: a Taiwan study," Applied Energy, vol. 88, no. 11, pp. 3816-3820, 2011.

[30] M. Meng, D. Niu, and W. Shang, " $\mathrm{CO}_{2}$ emissions and economic development: China's 12th five-year plan," Energy Policy, vol. 42, pp. $468-475,2012$.
[31] S. Liu, Y. Dang, Z. Fang, and N. Xie, Grey System Theory and Its Application, Science Press, Beijing, China, 2010.

[32] BP, "Statistical Review of World Energy 2013," http://www .bp.com/en/global/corporate/about-bp/statistical-review-of-worldenergy-2013/statistical-review-downloads.html.

[33] R. J. Hyndman and A. B. Koehler, "Another look at measures of forecast accuracy," International Journal of Forecasting, vol. 22, no. 4, pp. 679-688, 2006. 


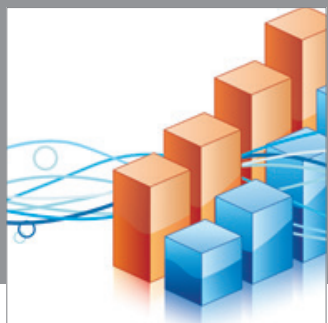

Advances in

Operations Research

mansans

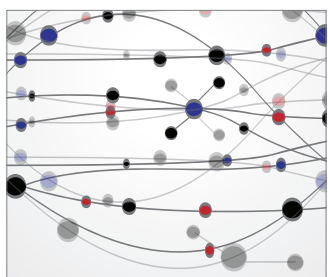

The Scientific World Journal
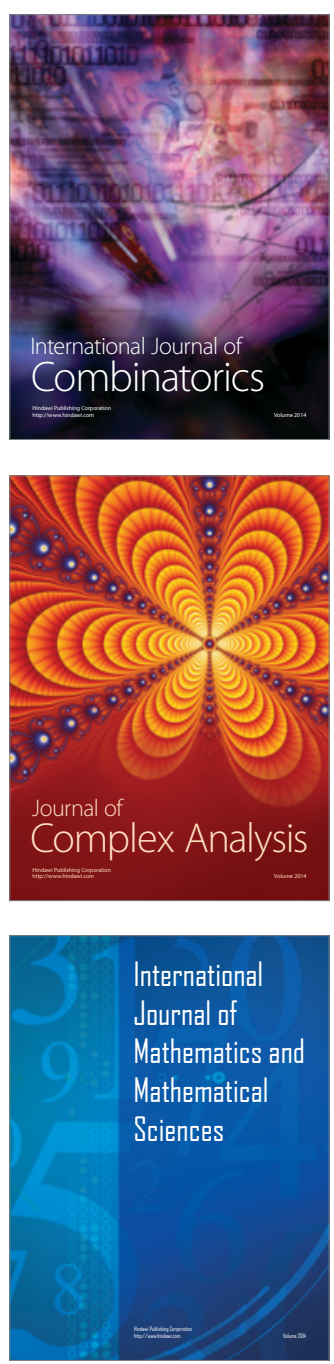
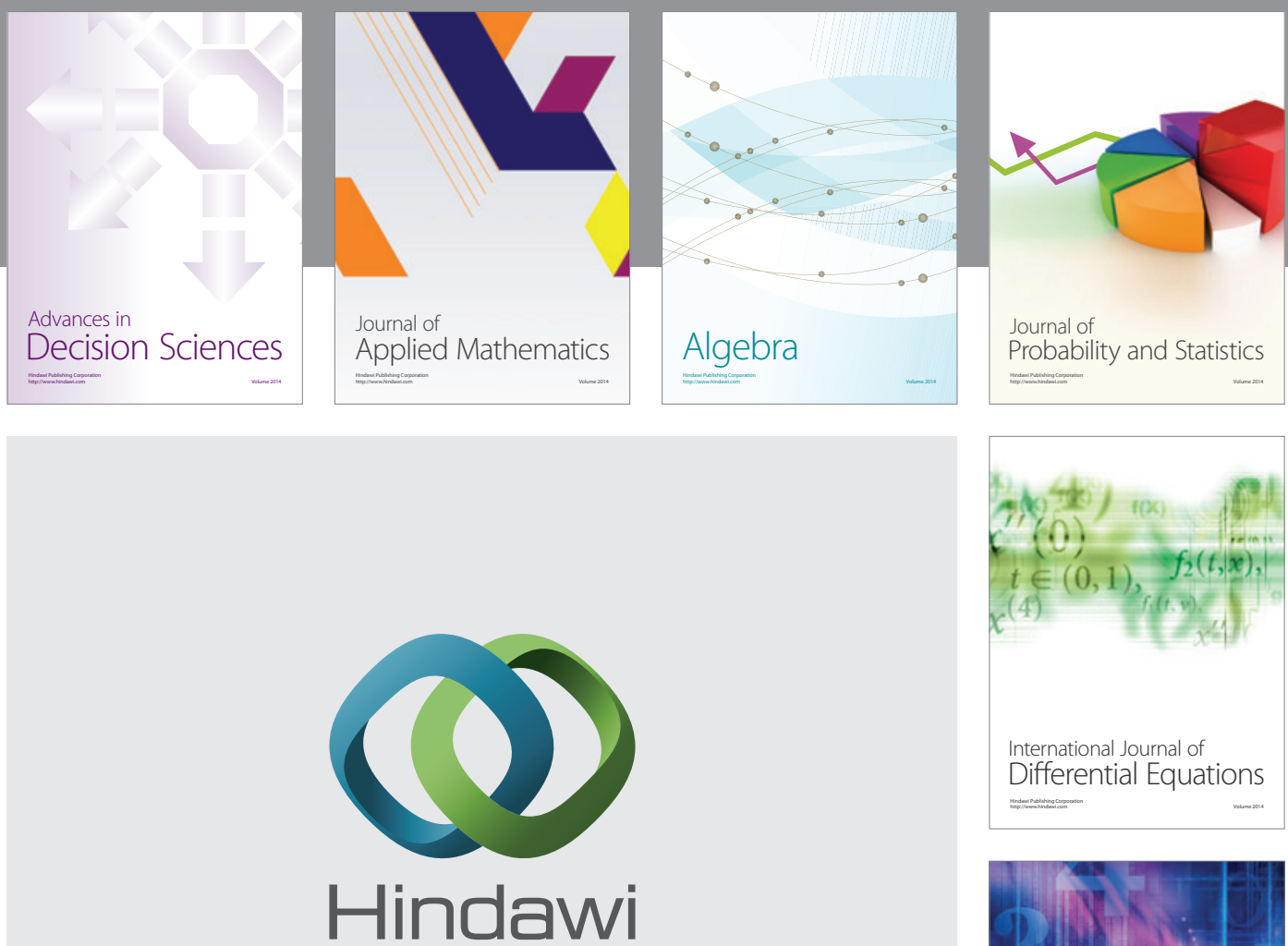

Submit your manuscripts at http://www.hindawi.com
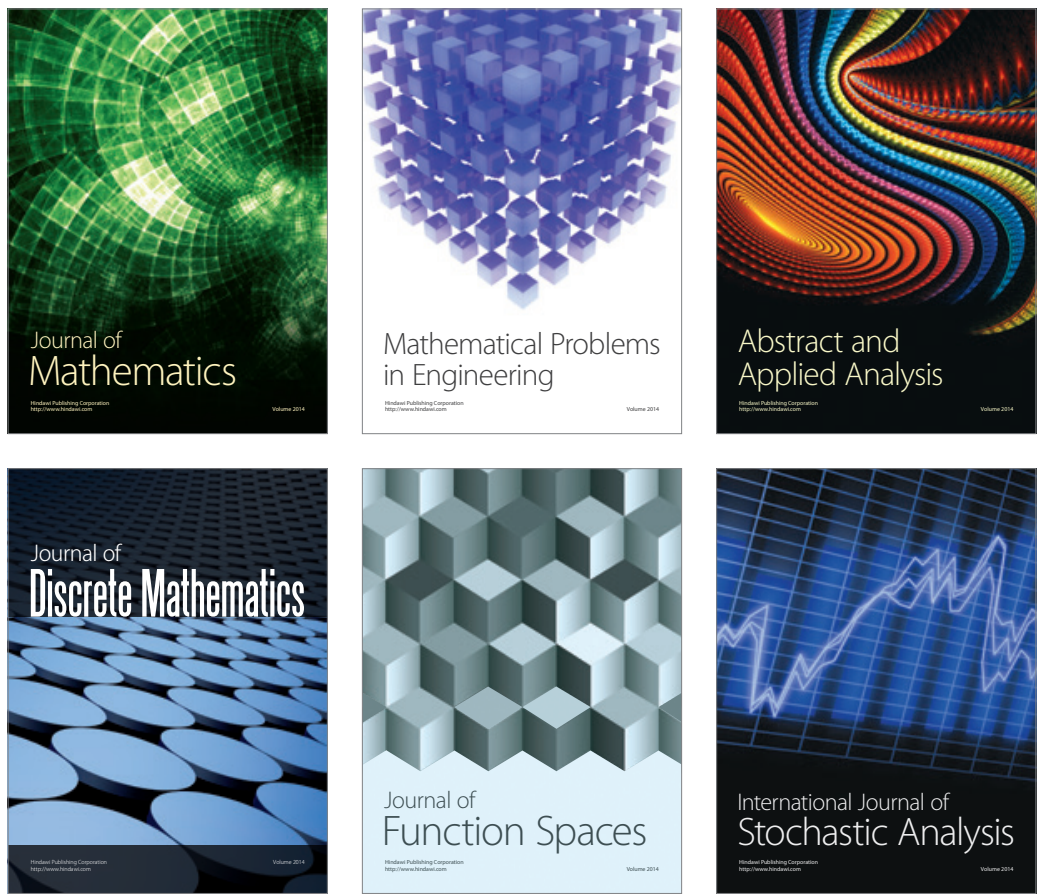

Journal of

Function Spaces

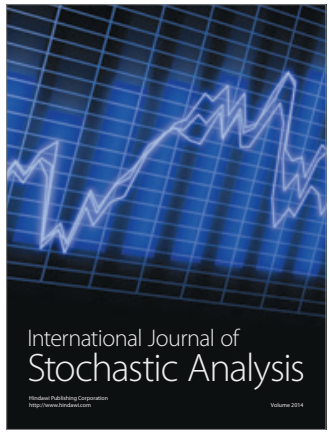

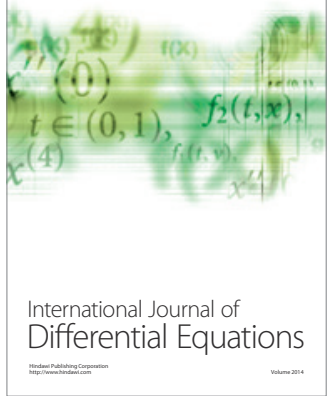
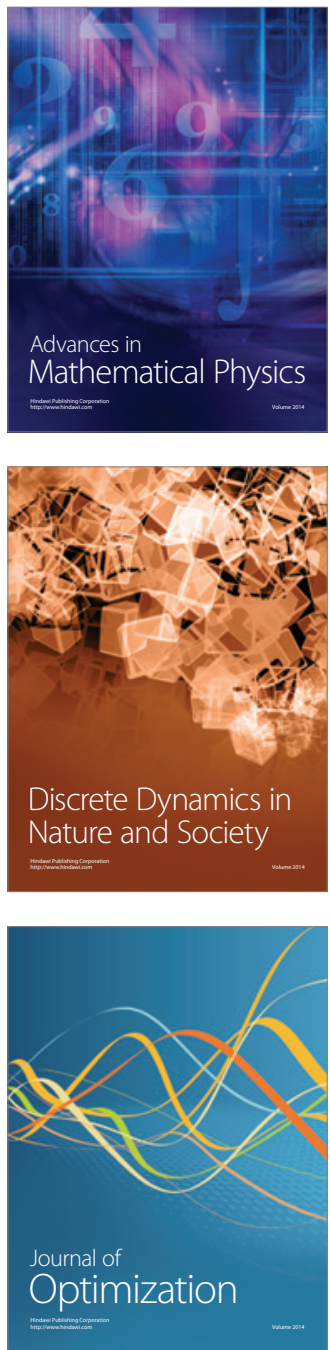